\title{
PLANNING A FIRST LEXICON: WHICH WORDS TO TEACH FIRST
}

\author{
Margaret Lahey \\ Montclair State College, Upper Montclair, New Jersey \\ Lois Bloom \\ Teachers College, Columbia University, New York, New York
}

\begin{abstract}
Various criteria are presented for selecting the first words to be taught to a child with a language disorder. In accordance with these criteria, recommendations are made for the inclusion of certain lexical items, the exclusion of other lexical items, and the organization of a first lexicon.
\end{abstract}

Clinicians make judgments about specific lexical items to teach a child learning a first language. In a recent article Holland (1975) presented some thoughts on the context and content of language therapy for children that can be considered as criteria for deciding which items should be included in a first lexicon. Her thoughts on context included using child language as the model to be taught, considering what is important to the child, using objects and events that are present and happening as nonlinguistic context for therapy, and stressing communication as a major goal of therapy. The article culminated with a core lexicon which reflected and was in accordance with these thoughts on context. The present article reflects further on establishing and organizing a first lexicon. These additional reflections integrate the issues discussed by Holland with three additional considerations-first, the ease with which a concept can be demonstrated in the context; second, the potential usefulness to the child of the words chosen as linguistic forms; and third, an organization of the lexical items according to the ideas that they code. The result is a set of suggestions for including certain items and excluding others in a first lexicon.

\section{LANGUAGE AND CONTEXT}

Language learning involves inducing the relationships among ideas of the world, linguistic forms, and communication. These three aspects of language have been referred to as content, form, and use of language by Bloom and 
Lahey (in press). The induction of the relationships among language content, form, and use is based upon the recognition of certain regularities-in the nonlinguistic context (as the recognition of similar objects in different contexts and the fact that these objects can disappear, reappear, be acted upon, located, and possessed), in the linguistic context (as the fact that words heard at one time are similar to words heard at another time), and in the social context (for example, that other persons can provide objects or attention). The child with a language disorder may have difficulty recognizing the regularities in any of these contexts (nonlinguistic, linguistic, social) or in inducing the relationships among the regularities. Thus the child may demonstrate weakness in knowledge of language content, form, or use or in the interrelationship between any two or among all three (Bloom and Lahey, in press).

Language intervention involves manipulation of the tangible nonlinguistic, linguistic, and social contexts in ways designed to enhance the induction of regularities within and between these contexts. The inductions that are the intended outcome of intervention can be specified as a set of goals which may be stated in terms of form, content, and use. While goals do not determine procedures, they do determine the types of nonlinguistic, linguistic, and social contexts that need to be included in intervention. Such goals focus on certain contexts in order to facilitate particular inductions but need not limit the child's language input or production to only these contexts.

Suggestions for a first lexicon form a set of goals for language learning. The lexicon presented here differs from the lexicon proposed by Holland (1975) primarily in terms of the way content and use are incorporated in the selection and organization of the forms of a lexicon. First, the selection of forms was based upon the ease with which the content they code can be demonstrated in the nonlinguistic context. Second, the selection of forms was based upon their usefulness in communication so that a child's potential use of the forms was considered. Finally, the lexicon is organized according to the regularities in the interaction between linguistic forms and the semantic content that such forms encode.

\section{Considering Nonlinguistic Context}

There is much to be learned about the factors that influence the clarity of the nonlinguistic illustration of concepts. For example, to facilitate the induction of the form slide as a means of referring to the act of going down a slide, should the context be (1) pictures of children sliding down a slide, (2) observation of children sliding down an actual slide, (3) the child himself sliding down an actual slide, or (4) some combination of all three? Leonard (1975) reported that observation of an actual demonstration was more effective than pictorial demonstration in teaching production of subject-verb relations. It is not clear whether observation of a demonstration is more or less effective in such learning than a child actually experiencing the action or state to be coded. 
Quite possibly the effectiveness of particular contexts varies with the child, the content to be coded, and the potential use of the forms being taught. While many children may be able to participate in certain actions at the same time that they attend to linguistic form, other children may become so involved in the act or participation that they cannot concurrently process linguistic form. For these latter children sequential presentation of form and participation (as "slide" spoken just before the child slides down) or observation of others acting may be more effective. While certain action relations, as sliding, may be easily demonstrated by others, such internal states as fear or hunger are more difficult to represent by picture or demonstration. Furthermore, certain functions of language can be taught in contexts that use pictures or demonstrations (such as labeling and regulating another's behavior), but other language functions require child participation (such as commenting on one's own actions and intentions or demands for services or objects). Thus the nonlinguistic context is influenced by the goals of language learning for a particular child. In like manner, the ease with which a concept can be illustrated in the nonlinguistic context should influence the goals of early language learning. Although empirical evidence is not yet available, it would seem that concepts which are difficult to demonstrate nonlinguistically should be presented after those concepts which can be more readily illustrated.

\section{Considering Linguistic Context}

In selecting the actual lexical items to be included as the goals of form in an intervention program, another consideration is the number of contexts in which the word can be used. Words that have broad application to many objects and events are words that will serve the child more frequently in his efforts to communicate and may offer more opportunities for input stimulation. The frequency with which the child may be able to use a word in his daily life should be an important consideration in choosing among lexical items.

Words used by young children can be dichotomized as substantive words or as relational words (Bloom, 1973). Substantive words refer to particular objects (as person and place names) or to categories of objects (such as chair and $d o g$ ). Relational words are words which refer to a relationship between objects and include parts of speech such as verbs, adjectives, and prepositions. The choice of particular substantive words will depend upon the child's environment and should refer to objects that will be frequently encountered. Labels for objects will not always be the most concrete nouns but rather the names that will be most useful to the child and perhaps most frequently heard-for example, car rather than Toyota, money rather than quarter, but apple rather than fruit (see Brown, 1958, for further discussion of this point).

Relational words are less specific than substantive words and may often be used to refer to many different objects. For example, no can be used to reject, deny, or note the disappearance of any object or event. Certain verbs are 
less object or event specific than others. Such verbs as give, get, make, and fall can refer to more objects than such verbs as eat and throw, and eat and throw are less specific than such verbs as drink or tear. The adjectives big and dirty can refer to more objects than can the adjectives orange or round. Thus, in selecting relational words to teach, one might consider first those relational words that are least object specific and that therefore have the most potential for communication in many different situations.

\section{CONSIDERING THE INTERACTION OF FORM AND CONTENT IN CHOOSING WORDS TO TEACH}

A lexicon can be best organized by the ideas the lexical items are intended to code, that is, by the content of language. The ideas that are commonly coded in early language have been described by a number of investigators (Bloom, 1970; Bloom, Lightbown, and Hood, 1975; Brown, 1973; Bowerman, 1973) and have been applied to teaching the language-disordered child by Miller and Yoder (1972, 1974), MacDonald and Blott (1974), and Bloom and Lahey (1972'; in press). These ideas can be categorized and used as the framework for a first lexicon.

The categories of content that language codes, also referred to as semantic relations or functions, can be viewed apart from the actual objects or events that are talked about. For example, action on objects can be considered as a category of the content of language, but while we all talk of actions on objects the specific action and the specific objects acted upon can vary with age, interest, and culture. Consequently one of us may talk about hitting golf balls, and another about riding horses or bikes. Each is an example of the content category of action. A list of certain categories that are common to adult language and found in the speech of young children is presented in the Appendix. The list is not exhaustive of all the relations found in child speech but represents the ideas coded most often by single-word utterances and early multiword utterances. The information in the Appendix comes from Bloom (1970, 1973) and has been partially presented in a somewhat similar form by Bloom and Lahey (1972'; in press) and Miller and Yoder (1974).

To complete the organization of a first lexicon some lexical items which can be used to code the ideas listed under content are suggested. These words include first, relational words that are not object specific. While a number of alternatives are listed in the first column, one item for each category may be used at first. Relational words that are more object specific are listed in the second column. Choices from and additions to this column can be made according to the child's interest. The last column includes space for substantive words. These words should be chosen to reflect the child's interest as well as the frequency with which the child will come in contact with particular

1L. Bloom and M. Lahey, A proposed program for teaching language to the language disordered child. Unpublished manuscript, Teachers College, Columbia Univ. (1972). 
people or objects. Holland (1975) has suggested particular toys for consideration (beads, blocks, ball, car, and doll). These are probably appropriate for many children, but we leave the selection open for the child who is interested in cats, bikes, airplanes, guns, or other objects.

The nouns that are taught can be included in many of the same contexts that illustrate the content categories. A child can reject by shaking his head and naming the object rejected ("cat" as he pushes the cat away). He can comment on or request the recurrence of an object by naming the object ("cat" as he points to one cat and "cat" as he points to another). Objects acted upon can be named as can the person acting or the place an object is located ("cat" as the child pats the cat, the cat scratches the chair, or the child puts a robe on the cat). In addition, the object that has disappeared can be named ("cat" as the child looks for the cat who has gone under the chair). A child can label an object ("cat" as he picks it up) or he can name the person or animal that is associated with an object ("cat" while pointing to the dish the cat eats from). Nouns can also be used to name objects or persons that the child is demanding or requesting ("cat" as he whimpers and tries to pull the cat from another child).

\section{Reasons for Excluding Certain Form/Content Interactions in a First Lexicon}

Certain content categories and certain forms that have not been included as yet may seem like they should belong in a first lexicon. One such category can be referred to as state--a category that includes coding of such internal states as happy, sad, hate, scared, love, hunger, or tired. A second category is affirmation-a category coded by a word as yes. Lastly, personal pronouns and lexical items which refer to particular attributes are not included. The reasons for not suggesting these categories and the use of these lexical items in a first lexicon are presented in the following sections.

Internal States. Two of the lexical items that Holland proposed and that are omitted here are hate and scared, both chosen by Holland (1975) because of their potential importance to the child. Holland's rationale was that emotions such as these are a central part of childhood and so should be a central part of language teaching. If such emotions are, in fact, a central part of a child's life, then the lexical items meet the criteria for teaching content that is important and relevant. Lexical items which code such emotional states offer other problems for teaching that need to be considered.

To learn a form to code an emotional state (as hate, love, scared, happy, sad) the state must be experienced by the child as the form is presented. The child must induce that it is a feeling that the word codes and not the objects or event that cause the state, nor the facial expressions, bodily postures, or gestures that accompany the state. It is interesting that in normal language development children talk of actions earlier and more frequently than they talk of states (Bloom, Lightbown, and Hood, 1975). While it is possible that emotional states are not as important to the young child as they are to the 
older language-disordered child, it is also possible that the normal sequence of development in young children can be partially accounted for by the difficulty a language learner has in making form/content inductions about concepts that are not externally tangible.

As Holland (1975) has suggested, therapy should focus on what is happening in the context. States happen inside. While they do have external manifestations, these manifestations are not the state. It is easier to teach the forms that code the manifestations of internal states than to teach forms that code the state itself. Thus kiss, a word chosen by Holland to code the emotional state of love, is a demonstration of love and is tangible-it happens, it can be seen and felt. A child can learn to talk about manifestations of an internal state (as "no" for the rejection of feared or hated objects and events), or learn to request comfort in the face of such situations (as the action hug or the person mommy). The words that code the state itself, however, might best wait for the learning of forms that code more tangible relationships between the child and an object or event (as recurrence-more; rejection-no; nonexistence-all gone; and identification or existence-there or object names) and relationships between objects (as action-kiss; attribution-big; and possession-Mommy car). These alternatives are in accordance with the thoughts which have been added here on ease of nonlinguistic illustration and the usefulness of words. If the meaning of hate is intended to be rejection (which may have been Holland's intention since she referred to it as "active verb" and used it as an example of syntactic coding of rejection), it too fits the criteria mentioned. To eliminate confusion with the adult meaning of hate as an internal state, the use of the alternate form no would be appropriate. Forms to code internal states are not included in the lexicon until after the child has begun syntactic coding of the categories listed in the Appendix.

Affirmation by "Yes". Should both yes and no be included in an early lexicon? Bloom and Lahey (in press) point out that most utterances are assertions about things that exist or happen-they are statements that are affirmations and therefore unmarked. It is not necessary to say "yes" unless someone questions the truth of a statement. We do not say, "Yes, I am hungry" unless someone asks, and if we say, "I am hungry" its truth is presumed. On the other hand, no is important as a device for marking the falsity of what would otherwise be an affirmation. No also communicates a number of ideas that are important to young children. For example, no is necessary to indicate a change from what is expected, as in the nonexistence or disappearance of an expected object or event ("There's no milk in the cup"); to code rejection of an object or event ("no wash"); or to prohibit an event ("no touch"). No then is not simply the opposite of yes.

The negative marker is also used to deny the truth value of another's statement (Statement: "It's hot in here." Response: "No, it's not.") or to answer a question (Question: "Are you hot?" Response: “No, I'm not."). This latter use of no or not to deny an affirmation or answer a yes/no question is similar to the use of yes, in that it too is used to respond to another's statement or to 
answer questions. One does not say, "No, I'm not hungry" anymore than one says, "Yes, I am hungry" unless asked.

Negation is important, then, because it serves to code notions that are not dependent upon being asked a yes/no question. It is necessary to communicate certain ideas even in the absence of such discourse. It is for this reason that no should be included in the first lexicon. A child affirms by making a statement and the only function of the lexical item yes is to answer a yes/no question or signal agreement with the statement of another. Thus the inclusion of yes is questionable.

There is a second objection to including yes in a first lexicon. Both yes to affirm and no to deny are dependent upon processing the utterance of another person. If a child has a vocabulary of less than 35 words and no knowledge of syntax, teaching yes is particularly difficult, because it depends upon linguistically processing a prior utterance. The only utterances that can probably be responded to by the child are related to identification of objects and actions already taught, for example, "Is that $x$ ?" This leads to interactions that are more like testing than teaching communication of ideas important to the child. Further, such interactions as "What's that?" and "Is that a ___?" stress the labeling of objects rather than the relationship of objects and events to each other and to the child. Thus yes has not been included as a form to be directly taught to the child in the very early stages of language learning.

Person Names vs Personal Pronouns. Four of the lexical items suggested by Holland (1975) are personal pronouns coding the speaker-hearer distinction ( $I$, me, or $m y$ in contrast to you and your). In addition, five items are person names (name of self, significant others, and the clinician). Recent research in language development (Bloom, Lightbown, and Hood, 1975; Nelson, 1975) has noted important differences among children in the extent to which they use pronouns. While some children use predominantly pronominal forms in their early syntactic utterances to code agents, objects, and location, other children use predominantly nominal forms (person, object, and place names) in the same type of utterances to code the same semantic relations. The child who refers to himself and others by name rarely uses the personal pronouns for the same function. By the time the children studied had a mean length of utterance (MLU) of 2.5 morphemes they used both pronominal and nominal reference for direct object, possessor, and place, with pronominal forms predominating for all children in the sentence subject position. Even at this point, however, the children rarely used the pronoun you. It is not yet clear why this difference in preference (nominal vs pronominal) exists or how to predict which form of reference a child will learn. Perhaps a first lexicon should include only one form of reference, nominal or pronominal, until the child's level of linguistic sophistication is near an MLU of 2.0 morphemes.

The teaching of nominal forms in the single-word utterance stage may be preferable for two reasons: personal pronouns are rarely found in the presyntax lexicons of children, and the shifting speaker/hearer distinction is a difficult one to teach. While most children learn pronouns which shift ref- 
erence from speaker to hearer without problems, it is difficult to design contexts that clearly illustrate this distinction to the language-disordered child. The child must learn that when speaking one calls oneself " $I$ " but when spoken to one is referred to as "you" or when spoken about one is referred to as "he" or "she." The problem is, of course, that the child never hears himself spoken of as "I." To take into account the child who may learn the distinction readily and prefer pronominal referents (for example, as the clinician washes she can say "Peggy wash," "I wash," and as the child washes "X wash," "you wash"). If the child learns the pronominal forms by this casual presentation, these forms can be stressed in the future. Thus direct teaching of personal pronouns is not recommended until the child's average length of utterance is 2.0 morphemes, and pronouns are not recommended for a first presyntactic lexicon.

Colors and Opposites. Although attribution is a category of content listed in the Appendix, note that there are no color words included within this category. Colors are easily illustrated in a nonlinguistic context and are thus often taught to and overemphasized with the language-disordered child. They are rarely found in the presyntactic or first syntactic lexicons of the normal child, nor do they have much value for communication. Colors are rarely mentioned by any of us unless we arc contrasting two identical objects. This use of attribution is not found in early child language. In addition colors do not have the broad topical reforence that some other adjectives have (for example, big and hot).

A second omission under adjectives is the polar opposites of the adjectives included. While big, heary, hot, and dirly are included, little, light, cold, and clean are omitted. The purpose of this omission is to encourage teaching each adjective before its oppositc. It has been observed that normal children tend to learn one adjective of a polar pair before another (Clark, 1970). Teaching opposites at the same time can confuse a child so that he rclates both hot and cold with any conception of heat, and heary or light with any conception of weight. While the opposite contrast may exist in the nonlinguistic context, it can be coded as nonexistence and attribution-for example, objects that are light can be referred to as "not heavy."

Thus the attributes that are taught first exclude colors and polar opposites. A few adjectives which can be used with many objects are included but attribution, in general, makes up a small part of the language used by young children and should not be overcmphasized. Other categories are more frequently used (Blom, Lightbown, and Hood, 1975) and are more communicative.

\section{CONCLUSION}

Once the clinician is prepared with an organizational structure of the content categories that make up the meaning relations to be taught and a small core of useful lexical items that code the content categories, the clinician can 
select other relational and substantive words to complete the matrix (which is in fact an outline of form-content goals), according to the interests and needs of the child. Forms are presented in contexts that illustrate the content categories, for example, acting on objects, locating objects, and rejecting objects. As the child observes or participates in these contexts the clinician provides relevant linguistic forms to code content. Goals for two-word utterances can be derived from combinations of the known forms in the same contexts, while new lexical forms are also gradually added. Before goals are extended to twoword utterances or new lexical items, however, the clinician encourages the child to use the words already learned both as comments on objects and relations between objects and himself in the environment and as a means of manipulating people and obtaining objects. Thus the initial goals include not only forms to talk of ideas (content) but the use of these forms as a means of communication before additional and more complex forms become goals.

This organization of a first lexicon provides flexibility, tends to remind the clinician that words are taught for meaning (form interacting with content), and stresses teaching words for communication (use) and not only as labels. In addition, this organization integrates the thoughts expressed by Holland (1975) (the use of child language as a model, consideration of what is important to the child, using objects and events that are present and happening as the focus of therapy, and stressing communication as the major goal of therapy), with the ideas added here (the ease with which the concept can be nonlinguistically expressed, the usefulness of the words chosen, and an organization of lexical items according to content categories). Lastly, the organization leads readily to developing goals for syntactic two-word utterances as found in normal language development and as reflected in the form-content goals suggested by Bloom and Lahey (19721; in press), Miller and Yoder (1972, 1974), and MacDonald and Blott (1974).

\section{ACKNOWLEDGMENT}

This paper was supported in part by National Science Foundation Grant Soc 74-24126 to Lois Bloom. Margaret Lahey is currently on the faculty of Hunter College of the City University of New York and is a research associate at Teachers College, Columbia University. Requests for reprints should be sent to Margaret Lahey, Department of Communication Sciences, Hunter College, 105 East 106th Street, New York, New York 10029.

\section{REFERENCES}

Bloom, L., Language Development: Form and Function in Emerging Grammar. Cambridge, Mass.: MIT Press (1970).

Bloом, L., One Word at a Time: The Use of Single-Word Utterances Before Syntax. The Hague: Mouton (1973).

Bloom, L., and LAhey, M., Language Development and Language Disorders. New York: Wiley (in press).

Bloom, L., Lightbown, P., and Hood, L., Structure and variation in child language. Monogr. Soc. Res. Child Dev., 40, (1975).

Bowerman, M., Early Syntactic Development: A Cross-Linguistic Study with Special Reference to Finnish. Cambridge: Cambridge Univ. Press (1973). 
Brown, R., How shall a thing be called? Psychol. Rev. 65, 14-21 (1958).

Brown, R., A First Language. Cambridge, Mass.: Harvard Univ. Press (1973).

CLARK, H., The primitive nature of children's relational concepts. In J. Hayes (Ed.), Cognition and the Development of Language. New York: Wiley (1970).

HollaND, A., Language therapy for children: Some thoughts on context and content. J. Speech Hearing Dis., 10, 514-523 (1975).

LEONARD, $L$., The role of nonlinguistic stimuli and semantic relations in children's acquisition of grammatical utterances. J. exp. child Psychol., 19, 346-357 (1975).

MacDonald, J. D., and Blotr, J. P., Environmental language intervention: The rationale for a diagnostic and training strategy through rules, context, and generalization. $J$. Speech Hearing Dis., 39, 244-256 (1974).

Muller, J., and Yoder, D., A syntax teaching program. In J. Mclean, D. Yoder, and R. L. Schiefelbusch (Eds.), Language Intervention with the Retarded. Baltimore: University Park Press (1972).

MILlER, J., and YODER, D., An ontogenetic language teaching strategy for retarded children. In R. L. Schiefelbusch and L. L. Lloyd (Eds.), Language Perspectives: Acquisition, Retardation, and Intervention. Baltimore: University Park Press (1974).

NELSON, K., Individual differences in early semantic and syntactic development. In D. Aaronson and R. Rieber (Eds.), Developmental Psycholinguistics and Communication Disorders. New York: New York Academy of Sciences (1975).

Received January 5, 1976.

Accepted July 23, 1976. 


\section{APPENDIX}

\section{Organization of a First Lexicon by Content and Form}

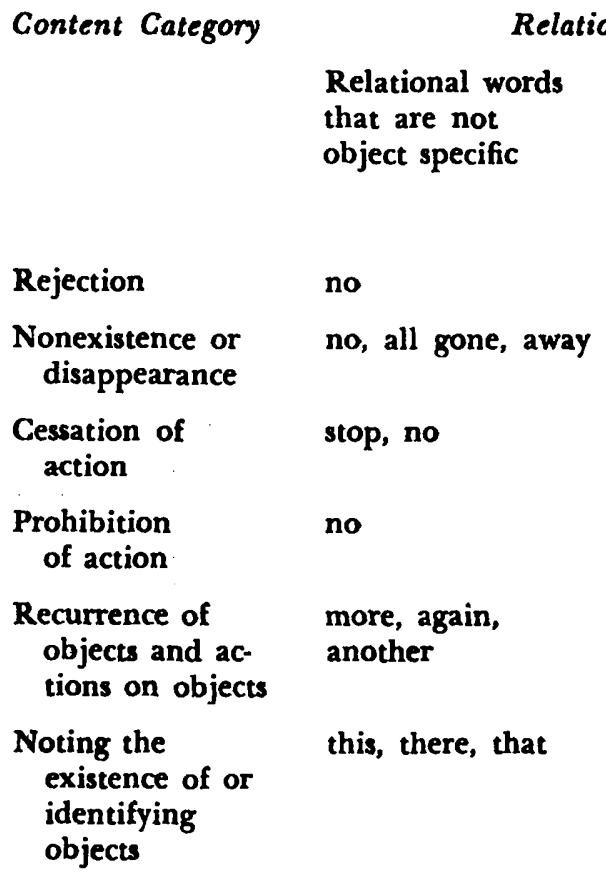

Actions on objects

give, do, make, get, throw, eat, wash, kiss
Actions involved in locating ob- jects or self

Form

Relational words that are more specific to objects but still relate to many objects

Substantive Words put, up, down, sit, fall, go

big, hot, dirty, heavy descriptions of objects

Persons associated with objects (as in possession) 